\title{
Le pôle territorial de coopération économique de Romans-sur-Isère dans la Drôme (France) : une organisation au service de la relance de la dynamique territoriale
}

\author{
Cécile Perret, maître de conférences, IUT de Chambéry \\ IREGE, Université de Savoie Mont Blanc \\ Membre collaborateur au CRDT, Université du Québec \\ Christiane Gagnon, professeur associé au département des sciences humaines \\ Université du Québec à Chicoutimi \\ Responsable de l'axe développement durable du CRDT
}

\section{INTRODUCTION}

La création, le renforcement et la qualité des liens sociaux qui dessinent de nouvelles configurations territoriales ainsi que le rôle de l'Économie Sociale et Solidaire (ÉSS) sont alors posés au cœur de la dynamique territoriale.

Le territoire, à la fois support et producteur d'identité(s) (Perret et Gagnon, 2016) ${ }^{1}$, peut être envisagé comme un construit social qui s'élabore à partir de la complexité des systèmes humains. Il peut aussi être envisagé comme un lieu cristallisant différentes configurations qui reposent sur la notion d'externalité formalisée par Alfred Marshall. Ce dernier montre que les économies d'échelles peuvent provenir d'effets externes générés par le contexte productif, d'une « atmosphère industrielle » (Pecqueur, 2007) $)^{2}$ et même industrieuse, propre à un territoire, que des entreprises ancrées territorialement construisent et dont elles bénéficient.

Face au déclin des districts industriels ${ }^{3}$, à une concurrence exacerbée parfois destructrice et à la désindustrialisation, la nécessité de renforcer les liens pour une économie plus solidaire, plus sécurisante et plus juste fait son chemin. L'innovation peut aussi être sociale ${ }^{4}$, répondant à des besoins fondamentaux et créant de nouvelles relations ou collaborations. La création, le renforcement et la qualité des liens sociaux qui dessinent de nouvelles configurations territoriales ainsi que le rôle de l'Économie Sociale et
Solidaire (ÉSS) sont alors posés au cœur de la dynamique territoriale.

À Romans-sur-Isère, le déclin du district industriel de la chaussure a largement modifié le paysage industriel de la ville dès la fin des années 80. De nombreuses entreprises ont ainsi délocalisé leur production à l'étranger ou ont fermé, obligeant les acteurs du territoire à imaginer de nouvelles solutions pour faire face à la précarité sociale (Perret, 2015) ${ }^{5}$. Ainsi, récemment, un pôle territorial de coopération économique (PTCE), le Pôle Sud Archer, et la Société par Actions Simplifiée (SAS) Groupe Archer, ont été créés, dessinant une nouvelle configuration territoriale qui allie économie sociale et solidaire (ÉSS), collaborations diverses, aide à la création et relocalisation d'activités économiques.

Notre hypothèse de départ, à valider, est que les nouvelles configurations territoriales, telle celle de PTCE, véritables « clusters d'innovation sociale à la française » (Pérard, 2015) ${ }^{6}$, et le socio-financement - aussi appelé financement participatif de proximité - interpelle une volonté d'implication citoyenne dans le développement territorial. Pour ce faire, nous avons réalisé plusieurs entretiens avec l'équipe dirigeante du Pôle Sud Archer, participé à l'assemblée générale mixte de la SAS Groupe Archer du 6 juin 2016 et à des animations au sein du PTCE. Nous avons également consulté divers documents concernant ce PTCE, en particulier sa charte et les statuts des entités qui le constituent. 
La première partie de cet article propose d'analyser en quoi les PTCE, qui s'inscrivent dans la logique de l'ÉSS, peuvent entraîner une nouvelle dynamique territoriale, voire se transformer en milieu innovateur générant à la fois un apprentissage social et une interaction avec le milieu. Nous avons ici adopté une approche abordant des aspects plus culturels souvent peu développés dans la littérature sur les milieux innovateurs généralement plus axée sur le monde de la production. La deuxième partie est consacrée à l'étude du cas du PTCE de Romans-surIsère qui stimule les pratiques collaboratives, notamment grâce à des animations économiques, intensifiant alors l'interaction entre les acteurs du territoire. La troisième partie présente, elle, le rôle du PTCE et de la finance de proximité dans la création et la relocalisation d'activités qui privilégient les circuits courts.

\section{LES PÔLES TERRITORIAUX DE COOPÉRATION ÉCONOMIQUE : DES AGENTS DE DYNAMIQUE TERRITORIALE INNOVANTE}

C'est la capacité d'une configuration territoriale, enracinée dans une culture locale, à générer des innovations, à se transformer en milieu innovateur qui soutient la dynamique territoriale.

\subsection{Le milieu innovateur : le rôle clef des acteurs et du territoire}

La notion de milieu innovateur (MI) émane d'une réflexion sur les sources de l'innovation (Darchen et Tremblay, 2008, 12) ${ }^{7}$; elle explore l'idée selon laquelle l'innovation serait contenue de façon latente dans certains territoires (Aydalot, 1986) ${ }^{8}$. Selon Richard $(2007,83)^{9}$, le MI symbolise un milieu «à succès », gage d'un territoire performant. Il se distingue, notamment, par des acteurs capables de générer «un processus collectif cognitif qui engendre un cadre, un climat, une atmosphère ou une culture au dynamisme social, politique, culturel, technologique, administratif et économique » (Proulx, 1994 cité par Richard, 2007, 83). Le territoire est donc au centre de cette notion de MI.

Le territoire en MI peut être envisagé comme un espace vécu (Frémont, 1972) intégrant les dimensions culturelles et sociales portées par les acteurs. Il peut aussi être vu sous l'angle, d'une part, d'un support identitaire pour les pratiques d'acteurs (Bassand, 1982) ${ }^{10}$ et, d'autre part, comme un producteur d'identité par le biais de ces mêmes pratiques (Perret et Gagnon, 2016). Ce territoire identitaire, avec les valeurs ou croyances des acteurs et leurs capacités à collaborer ${ }^{11}$ ou à entrer en lien les uns avec les autres, vont alors engendrer des modes de gou- vernance territoriale spécifiques et de pratiques socio-spatialisées.

De là, des configurations territoriales plus ou moins aptes à générer l'innovation peuvent techniquement se comprendre, selon Crevoisier $(2001)^{12}$, comme le résultat de l'articulation des ressources de l'entreprise et de son environnement. Le MI pourrait alors être défini de façon dynamique, comme «un ensemble d'acteurs territorialisé [et enraciné culturellement], acteurs qui réalisent des apprentissages multilatéraux et génèrent des externalités locales spécifiques à l'innovation » (Crevoisier, 2006, 62) ${ }^{13}$. Cette dynamique d'acteurs peut également déboucher, selon certaines conditions, sur des formes de plus en plus performantes de gestion en commun des ressources (Maillat et al., 1993 cité par Crevoisier, 2006, 62) et d'apprentissages.

\subsection{Le milieu innovateur comme lieu d'apprentissage}

La logique d'apprentissage apporte une dimension plus dynamique au milieu et elle souligne l'importance d'éléments tels que le savoir-faire, la culture technique, le niveau de qualification et de compétences (Coppin, 2002, 33) ${ }^{14}$.

Les apprentissages, qui génèrent des externalités locales spécifiques à l'innovation, vont dépendre : 1) de l'interaction des acteurs avec le milieu, i.e. de la capacité des acteurs à entrer en lien les uns avec les autres et à activer ces liens ${ }^{15}$ et 2) de la capacité des acteurs à intégrer de nouveaux savoirs découlant de leur apprentissage du milieu (Darchen et Tremblay, 2008, 21-23). 
Les différentes configurations territoriales, tel le milieu innovateur, peuvent être caractérisées selon ces deux critères. Le MI représente une situation idéale vers laquelle toutes les autres configurations territoriales devraient évoluer pour peu qu'elles soient capables de renforcer leur interaction avec le milieu et leur capacité à intégrer de nouveaux savoirs. Le MI présente en effet, selon Darchen et Tremblay (2008), un fort degré d'apprentissage et une forte interaction avec le milieu, permise et renforcée par la culture locale. Sa finalité est de se renforcer en générant une véritable culture de l'innovation, support de la dynamique territoriale.

\subsection{Les pôles territoriaux de coopération économique et l'économie sociale et solidaire}

\section{Un PTCE répond à une logique collaborative et de mutualisation, de coopération ou de partenariat entre divers acteurs au service de projets économiques et sociaux innovants.}

À l'instar d'Hervé Defalvard (2015) ${ }^{16}$, l'ÉSS est ici envisagée comme un autre mode d'entreprendre et comme «un mode de développement économique, reposant sur les valeurs de solidarité et de durabilité, voire de sobriété; encourageant la création des pôles territoriaux de coopération économique $[\ldots]$ ». Effectivement, certains territoires ont concentré des organismes relevant de l'ÉSS au sein de véritables pôles, les PTCE, définis en France par la Loi relative à l'ÉSS ${ }^{17}$ du 31 juillet 2014 Article 9. ${ }^{18}$ Un PTCE répond à une logique collaborative et de mutualisation, de coopération ou de partenariat entre divers acteurs au service de projets économiques et sociaux innovants. Sa définition est ouverte car chaque territoire met en relation des acteurs différents en fonction de sa réalité économique et sociale. L'émergence d'une configuration territoriale particulière repose sur des pratiques elles-mêmes sous-tendues par des valeurs qui peuvent être plus ou moins exacerbées selon les territoires (Perret et Gagnon, 2016).

De nombreux organismes de l'ÉSS font appel, lors de leur création, à des financements dits solidaire et de proximité. Ces financements peuvent prendre la forme d'un socio-financement. Il recouvre 1) le don, 2) la récompense, préachat ou cadeau, 3) le prêt ou 4) l'investissement au capital des entreprises. Des plateformes spécialisées permettent la mise en relation directe d'investisseurs potentiels et de créateurs d'entreprises ou de porteurs de projets.

L'un des avantages de ce type de financement est qu'il permet à la fois une « étude de marché amont » du projet puisque les contributeurs soutiennent un produit à vendre ou une entreprise à créer, et qu'il permet aux citoyens de soutenir un développement local en participant au financement d'activités sur leur territoire. Les consommateurs deviennent alors de véritables consommacteurs. Cette notion recouvre, notamment, des aspects de responsabilité sociale du consommateur-citoyen capable, grâce à ses choix d'achat, de peser sur l'offre des producteurs et, donc, de devenir un véritable acteur du développement territorial. Selon Glémain, Bioteau et Artis $(2010,213)^{19}$, l'importance croissante des finances solidaires, définies comme «l'ensemble des interventions en haut de bilan, en garantie et, les opérations visant à faire accéder au financement de projets personnels et professionnels des individus en marge des marchés bancaires habituels ». Dans le point suivant, nous exposons comment le PTCE Sud Archer fait appel à la finance de proximité.

\section{LE RÔLE DU PTCE SUD ARCHER DANS LE REGAIN DE L'ACTIVITÉ ROMANAISE}

Ce rôle est développé sous l'angle de la genèse de la naissance du pôle et de ses axes d'action actuels.

\subsection{La genèse de la naissance du PTCE Sud Archer}

Archer est une association née à Romans dans les années 80, de la volonté d'acteurs locaux de tenter de trouver des solutions à l'exclusion et au chômage. Elle était destinée, à l'origine, à faciliter le retour de chômeurs à l'emploi, notamment au travers d'actions de formation. Mais, comme l'explique son cofondateur, actuel Directeur du Pôle Sud Archer, «en période de fort chômage, on a tendance à former des gens à des métiers... qui n'existent pas. D'où l'idée [...] de nous mêler de développement économique local, de créer de l'emploi, seule vraie issue à l'insertion ${ }^{20}$.

Le PTCE en lui-même est née de la volonté de la Chambre Régionale de l'ÉSS Rhône-Alpes 
(CRESS) et du Groupe Archer de fédérer des acteurs du bassin romanais pour conduire des actions de développement économique. Dans un premier temps, un comité local de la CRESS a été constitué et, en avril 2007, lorsque le Groupe Archer s'est trouvé en situation d'emménager dans un nouveau siège situé en zone industrielle de Romans, il a été proposé aux acteurs de l'ÉSS de les rejoindre pour constituer un pôle.

Aujourd'hui, le Groupe Archer, qui est au cœur du PTCE, est composé de 5 entités :

1) l'Association Archer qui propose des services à la personne,

2) Archer entreprises ${ }^{21}$ qui concerne a) le transport, la logistique et la distribution, b) les espaces verts, c) le BTP, d) la sous-traitance industrielle et e) la fabrication de chaussures (Atelier cuir et marque de chaussures Made in Romans).

3) Archer Intérim qui assure de la délégation de personnel,

4) Arcoop, dont l'activité principale est l'accompagnement à la création d'entreprise,

5) la holding d'animation SAS Groupe Archer immatriculée en 2007.

La SAS Groupe Archer compte à ce jour 112 actionnaires, principalement de Romans et ses alentours. Ce sont des particuliers, dont certains sont des salariés d'Archer issus du milieu associatif, des chefs d'entreprise, des élus locaux, des associations et d'autres acteurs. Les prises de participation au capital vont de $300 €$ à $150000 €$ pour le Comptoir de l'Innovation ${ }^{22}$. Il s'agit donc en partie d'un financement de proximité. Afin que d'autres actionnaires puissent s'y agréger plus facilement, il a été décidé, lors de son Conseil d'administration du 6 juin 2016 de la transformer en SAS à capital variable. Les éventuels dividendes sont limités au taux d'un livret d'épargne classique, les actionnaires étant plus préoccupés par le développement de leur territoire que par des gains financiers. Cette « entreprise de territoire » pense le développement économique à la fois pour l'organisation et pour le territoire d'accueil ${ }^{23}$. Elle est particulièrement sensible aux questions liées au renouveau productif, soit les relocalisations, les reprises d'entreprise, les coopérations industrielles ou les circuits courts de production et de distribution.
Cette « entreprise de territoire » pense le développement économique à la fois pour l'organisation et pour le territoire d'accueil.

Des structures associatives de microcrédit et de garantie solidaire telles que L'Adié ${ }^{4}$ ou le Fonds local France active, une société financière solidaire comme la $\mathrm{Nef}^{25}$, le réseau COORACE ${ }^{26}$, l'Union Régionale des $\mathrm{SCOP}^{27}$ \& $\mathrm{SCIC}^{28}$ Rhône-Alpes et un organisme de formation s'y agrègent alors. Le PTCE Sud Archer dans son ensemble est, bien évidemment, soutenu financièrement des organismes publics nationaux, régionaux ou locaux ${ }^{29}$. Pôle Sud Archer est également lauréat de l'appel à projets ${ }^{30}$ pour le développement des PTCE en 2014 avec pour thématique «Activité industrielle locale, insertion par l'activité économique ». Une enveloppe globale de trois millions d'euros a été répartie entre 23 projets lauréats.

\subsection{Les axes d'action du PTCE}

Pôle Sud Archer propose deux axes d'actions en sus de celui visant à lutter contre l'exclusion sociale : l'un qui permet d'améliorer l'apprentissage du milieu et l'autre qui permet d'approfondir l'interaction avec le milieu.

\subsubsection{Des actions favorisant l'apprentissage $d u$ milieu}

Pôle Sud Archer est un lieu d'échanges et de rencontres informelles ou formelles entre les acteurs locaux. Une soirée «Start up de territoire » a ainsi été organisée le 2 février 2016. Il s'agissait, durant la soirée, grâce à un jeu de stimulation de la créativité, de faire imaginer les entreprises de demain à quelque 250 acteurs locaux et fervents du développement local. À l'issue de cette soirée, chacun de participants indiquait ce qu'il pouvait apporter aux entreprises ainsi imaginées : mise à disposition de locaux, connaissances techniques spécifiques, connaissances relatives à la création d'entreprise... la capacité des acteurs à intégrer de nouveaux savoirs était alors mise en jeu.

L'équipe d'Archer vise, finalement, à faire de Romans un territoire expérimental et accompagnateur des futures entreprises

françaises qui privilégient le local, les circuits courts et le durable. 
Ces projets d'entreprises sont actuellement évalués et accompagnés par l'équipe dirigeante du PTCE afin d'en concrétiser certains. Des jeunes talents, comme par exemple deux étudiants de l'École Nationale Supérieure de Création Industrielle qui ont un projet original de fabrication de machine à laver le linge durable ${ }^{31}$ ont ainsi été accueillis par l'équipe du PTCE suite à cette soirée afin d'évaluer de quelles expertises ou réseaux l'équipe d'Archer pourrait leur faire bénéficier. L'École de l'entrepreneuriat ${ }^{32}$ du PTCE soutient également cette dynamique. Un site internet «Start up de territoire » a également été créé afin de permettre, à terme, une mutualisation des expériences avec d'autres territoires français. Enfin, un projet de fonds d'investissement pour les "start up de territoire » est en gestation. L'équipe d'Archer vise, finalement, à faire de Romans un territoire expérimental et accompagnateur ${ }^{33}$ des futures entreprises françaises qui privilégient le local, les circuits courts et le durable.

\subsubsection{Des animations économiques intensifiant l'interaction entre acteurs du territoire}

Des actions d'animation économique sont également réalisées par des associations actives au sein du PTCE et permettent d'intensifier les interactions entre les acteurs du territoire. Par exemple, l'Association entreprise Romans Bourg de Péage (ERB) regroupe une centaine d'entreprises et propose un certain nombre de prestations auprès de ses adhérents : travail sur les achats, mise en place de services communs, etc. Le lien créé entre les entrepreneurs peut alors devenir du capital social au sens de Bourdieu (1980), car la ressource peut se transformer en actif.

Un autre exemple est celui de l'Association Romans cuir, née en juin 2012. Elle vise à créer des synergies pour consolider les acteurs de la filière cuir. Elle mène une réflexion afin d'accentuer les dynamiques collaboratives, notamment sur la distribution des produits. Romans cuir qui a récemment lancé le label Véritable chaussure de Romans. Ce label exige que la découpe du cuir, le piquage et le montage des chaussures soient réalisés dans l'agglomération de Romans-sur-Isère.

Enfin, la Coopérative d'Activités et d'Emplois ${ }^{34}$ travaille particulièrement sur les coopérations locales en regroupant les compétences de ses entrepreneurs et propose des collaborations avec les entreprises présentes au sein du PTCE ou sur le territoire.

\section{Des actions d'animation économique sont également réalisées par des associations actives au sein du PTCE et permettent d'intensifier les interactions entre les acteurs du territoire.}

\section{LA CRÉATION D'ACTIVITÉS GRÂCE AU PTCE DE ROMANS-SUR-ISÈRE}

En 2015, le Groupe Archer représentait 1262 salariés, dont 126 salariés dans au moins 2 structures, faisant d'Archer l'un des employeurs les plus importants de la Drôme. Les caractéristiques sociales de ces employés sont : $59 \%$ sont des femmes, $36 \%$ relèvent des minima sociaux, $19 \%$ sont des jeunes de moins de 26 ans et $53 \%$ sont sans qualification ${ }^{35}$.

C'est autour d'acteurs du Pôle Sud Archer et de l'entreprise Veyret Techniques Découpe (VTD) ${ }^{36}$ qu'a émergé, en octobre 2008, un projet d'atelier de chaussures à Romans : Made in Romans. Cette création a été permise grâce à la SAS groupe Archer qui a acheté une ligne complète de fabrication de chaussures. Made in Romans a fait le pari de proposer des chaussures fabriquées dans la ville en créant un atelier cuir. En dehors des semelles, fabriquées à Cholet, plus de $90 \%$ des cuirs et des matières premières utilisées sont achetées dans un rayon de 20 kilomètres autour de Romans-sur-Isère. Elle effectue aussi de la sous-traitance pour des marques connues et fabrique, à la demande, des petites séries pour des stylistes ou des défilés. Elle a aussi lancé sa propre collection de chaussures, baptisée Made in Romans et possède un magasin d'usine. En 2015, l'atelier comptait huit salariés, dont d'anciens ouvriers de Charles Jourdan et de Stéphane Kélian, autrefois entreprises fleurons de la ville. Si l'activité chaussures est à l'équilibre depuis 2015, l'intérêt de cet atelier est surtout de montrer qu'il est encore possible de produire de la chaussure à Romans et d'attirer d'autres 
entreprises de la chaussure afin d'enclencher une dynamique de milieu innovateur ${ }^{37}$.

Quelques petites entreprises de fabrication de chaussures très spécialisées ont également été récemment crées à Romans. Milémil ${ }^{38}$, entreprise fondée en mai 2014, en fait partie. Elle propose des chaussures de football en cuir pleine fleur et coton biologique. Ses chaussures sont produites dans l'atelier cuir d'Archer. Elle a financé le démarrage de son activité sur la plateforme de don Ulule ${ }^{39}$. Elle a ainsi collecté 11206 euros grâce aux contributions de 115 personnes. Dans les commentaires apparents sur le site Ulule, il est clair que les investisseurs sont motivés par une fabrication française. La jeune marque 1083 propose, elle, des jeans en pièces détachées à coudre soi-même et des baskets. Tous ses produits ou matières premières sont fabriqués à moins de 1083 kilomètres de Romans ${ }^{40}$. Pour financer leur entreprise, les fondateurs ont, eux aussi, eu recours à la plateforme Ulule pour constituer leur capital de départ car «les banques ne financent pas la constitution de stocks, surtout en ce moment $\gg^{41}$. En quelques semaines, la collecte a permis d'enregistrer plus de 1400 précommandes, soit environ 160000 euros de chiffre d'affaires prévisionnel. Avec cette somme, qui a permis d'acheter les fournitures, les

\section{CONCLUSION}

Le PTCE Archer répond à une logique collaborative et de mutualisation pouvant former un écosystème coopératif. Il peut devenir un véritable milieu innovateur si son apprentissage du milieu et son interaction avec le milieu sont suffisamment forts pour rendre le territoire attractif et enclencher une dynamique territoriale. Suite à la recherche documentaire, aux observations et entrevues, il apparait qu'au moins trois indices vont dans ce sens :

\section{Le PTCE Archer répond à une logique collaborative et de mutualisation pouvant former un écosystème coopératif.}

1) Le PTCE Sud Archer de Romans-sur-Isère, animé par une équipe dirigeante dynamique, pragmatique et concernée par l'avenir de son territoire, est créateur de liens et d'activités tout en luttant ainsi contre l'exclusion sociale. La premières séries ont pu être fabriquées. Un autre gros projet autour de la chaussure a également utilisé le socio-financement: la plateforme Le Soulier Français ${ }^{42}$ qui vient de collecter 500000 euros via la plateforme de sociofinancement Raizers.

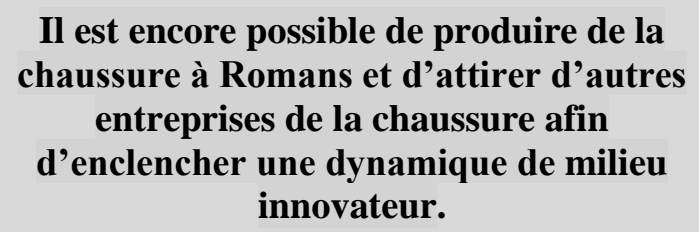

Il est encore possible de produire de la chaussure à Romans et d'attirer d'autres entreprises de la chaussure afin d'enclencher une dynamique de milieu innovateur.

Milémil a ouvert un magasin au centre de Romans en 2014. Depuis fin 2015, le magasin présente non seulement des chaussures Milémil mais également Made in Romans, Soft-In by Insoft ${ }^{43}$, Max Vincent ${ }^{44}$ ainsi que de la maroquinerie artisanale Escarcelle ${ }^{45}$. Les charges du magasin et le salaire de la vendeuse sont mutualisés ${ }^{46}$. L'atelier Max Vincent propose, lui aussi, à la vente des chaussures Made in Romans dans son magasin d'usine. Une logique collaborative est ainsi à l'œuvre. Un ambitieux projet visant à créer une sorte d'éco-parc pour une production labellisée est à ce jour envisagé par les dirigeants du Groupe Archer ${ }^{47}$.

création de liens entre les acteurs du territoire engendre à la fois du sens et des opportunités économiques : l'Association entreprise Romans Bourg de Péage réalise des commandes groupées pour une centaine d'entreprises, Romans cuir permet de créer des synergies pour consolider la filière cuir, la SAS Groupe Archer réinsère des personnes au chômage ou en difficulté, etc.

2) La SAS Groupe Archer pense le développement économique à deux niveaux : pour son organisation et pour le territoire sur lequel elle est implantée. Des citoyens participent à son capital et, de fait, s'impliquent dans le développement de leur territoire. Cette participation citoyenne pourrait à l'avenir se renforcer grâce à son nouveau statut de SAS à capital variable. La SAS participe à des formes de coopération et de partenariat qui permettent de développer 
l'activité économique du lieu d'implantation grâce à la relocalisation d'activités, telles que l'atelier MGI Coutier ou la création d'activités comme l'Atelier cuir; tout en inventant de nouvelles formes d'organisation et en aidant de jeunes talents à créer leur « start up de territoire ».

3) La finance solidaire et participative joue un rôle dans le regain de dynamisme du territoire. La possibilité de réaliser des collectes de fonds via des plateformes de socio-financement permet à la fois de lancer de nouvelles entreprises ou d'en renforcer d'autres, notamment quand les banques restent « frileuses » face à de jeunes créateurs. En investissant via les plateformes, les citoyens signalent leur intérêt pour la fabrication d'un produit particulier, d'un produit fabriqué en France ou sur un territoire particulier. Ils deviennent alors de véritables consommacteurs qui participent pleinement au développement de leur territoire.

Le renforcement de cette tendance pourrait bien être le signe d'une volonté d'implication des citoyens dans le développement territorial, dans un contexte de crise économique et de perte de confiance dans «le politique » en général. Le PTCE contribue à redynamiser un territoire fragilisé depuis les années 80 par la disparition de la mono-industrie de la chaussure et à renforcer les capacités des acteurs en vue de la maîtrise sociale du développement de leur territoire et l'inclusion de tous ses habitants.

\section{BIBLIOGRAPHIE ET NOTES}

${ }^{1}$ Perret, Cécile et Gagnon, Christiane (2016). «Identité(s), gouvernance villageoise et développement territorial viable en Kabylie », CIST, $3^{\text {e }}$ colloque international. En quête de territoire(s)? Looking for territories? Proceedings, 17-18 mars 2016, Cité des territoires (IGA+IUG) Grenoble, p. 377-383.

${ }^{2}$ Pecqueur, Bernard (2007). «L'économie territoriale : une autre analyse de la globalisation », L'Économie politique 1/ 2007 (no 33), p. 41-52.

${ }^{3} \mathrm{Ce}$ type de région industrielle doit son succès à un mélange d'émulation, de coopération et de concurrence entre PME spécialisées dans des savoir-faire traditionnels (Pecqueur, 2007).

${ }^{4}$ L'innovation sociale porte aussi bien sur la finalité que sur les processus, notamment sur la gouvernance (Pérard, 2015, 5). Pérard, Pierre (2015). Les clusters d'innovation sociale. Analyses et bonnes pratiques européennes, Collection « Notes d'analyse » dirigée par Denis STOKKINK / Juin 15, Économie sociale, p. 5.

${ }^{5}$ Perret, Cécile (2015). « Du district industriel au pôle territorial de coopération économique. La chaussure de Romans-sur-Isère vers un renouveau productif? », session pour l'Institut des Hautes Études de Développement et d'Aménagement des Territoires en l'Europe (IHEDATE), 19 novembre 2015 à Romanssur-Isère. URL : https://halshs.archives-ouvertes.fr/ halshs-01186435/.

${ }^{6}$ Pérard, P. (2015). Les clusters d'innovation sociale. Analyses et bonnes pratiques européennes, Collection « Notes d'analyse » dirigée par Denis Stokkink / Juin 15, Économie sociale, 19 p.

${ }^{7}$ Darchen, Sébastien et Tremblay, Diane-Gabrielle (2008). «Les milieux innovateurs et la classe créative : revue des écrits et analyse de leur application en milieu urbain ", Note de Recherche de la Chaire de Recherche du Canada sur les enjeux socio-organisationnels de l'économie du savoir.

${ }^{8}$ Aydalot, P. (dir.) (1986). « Trajectoires technologiques et milieux innovateurs », Milieux innovateurs en Europe, Èd. Groupe de recherche européen sur les milieux innovateurs, Paris, p. 347-361.

${ }^{9}$ Richard, Guillaume (2007). « Très petite entreprise, réseau d'innovation et milieu innovateur : le cas de la région Rhône-Alpes », Staps 1/2007 (no75), p. 83-100.

${ }^{10}$ Bassand, M. (1982). Maldéveloppement régional et identité : pour un développement endogène, Lausanne, Presses polytechniques et universitaires romandes.

${ }^{11}$ «L'objet de la collaboration [étant] de créer une vision partagée et des stratégies articulées pour faire émerger des intérêts communs dépassant les limites de chaque projet particulier » (Chrislip, 2002 cité par LE ROUX, Serge $(2007,191)$. « La mise en œuvre d'une approche collaborative comme facteur d'innovation dans les PME-PMI », Marché et organisations 2/2007, $\mathrm{n}^{\circ} 4$, p. 189-208.

${ }^{12}$ Crevoisier, Olivier (2001). « L'approche par les milieux innovateurs : état des lieux et perspectives », Revue d'Économie Régionale et Urbaine, $\mathrm{n}^{\circ} 1$, p. 153-166.

${ }^{13}$ Crevoisier, Olivier (2006). « Économie régionale, économie territoriale : la dynamique des milieux innovateurs », in Mollard, A, Sauboua, E., HIRCZAK, M. (2006). Territoires et enjeux du développement régional, p. 61-82.

${ }^{14}$ Coppin, Olivier (2002). «Le milieu innovateur: une approche par le système », Innovations 2/2002 ( $\left.\mathrm{n}^{\mathrm{0}} 16\right)$, p. 29-50.

${ }^{15}$ L'activation des liens créés peut être analysée à la lumière du concept de capital social au sens de Bourdieu (1980) qui le considère en tant qu'ensemble de ressources actuelles ou potentielles qui sont liées à la possession d'un réseau durable de relations. Ces ressources peuvent être transformées en actifs 
(Bourdieu, Pierre (1980). « Le capital social. Notes provisoires », Actes de la recherche en sciences sociales, vol. 31, janvier, p.2-3).

${ }^{16}$ « Hervé Defalvard : «L'économie sociale et solidaire est l'alternative au néolibéralisme»», entretien réalisé par Vittorio De Filippis, 2 novembre 2015, Libération.

${ }^{17}$ Dans son article 5 , cette loi vise à reconnaître les PTCE et à soutenir leur développement.

${ }^{18}$ Un PTCE est un « regroupement sur un même territoire d'entreprises [ de l'ESS...] qui s'associent à des entreprises, en lien avec des collectivités territoriales et leurs groupements, des centres de recherche, des établissements d'enseignement supérieur et de recherche, des organismes de formation ou toute autre personne physique ou morale pour mettre en œuvre une stratégie commune et continue de mutualisation, de coopération ou de partenariat au service de projets économiques et sociaux innovants, socialement ou technologiquement, et porteurs d'un développement local durable ».

${ }^{19}$ Glémain, Pascal, Bioteau, Emmanuel et Artis, Amélie (2010). «Finances solidaires et territoires : analyses en Bretagne et Pays-de-la-Loire ", Revue d'Économie Régionale \& Urbaine, 2/2010 (mai), p. 213-233.

${ }^{20}$ Source : http://www.info-economique.com/en-une/aromans-1-entrepreneuriat-au-service-du-territoire-459.

${ }^{21}$ La première société créée à l'initiative d'Archer a vu le jour en 1992. Il s'agissait d'Arbati, dans le secteur Bâtiments et Travaux Publics (BTP) et qui a été liquidée en 2014. Vint ensuite Arcoop, créée en 2007, et qui assure un accompagnement à la création d'entreprise.

${ }^{22}$ Le Comptoir de l'innovation investit, au travers de ses fonds, dans des activités et des entreprises variées collaboratives, circulaires, sociales - qui ont en commun la recherche d'un fort impact social et/ou environnemental.

${ }^{23}$ Entretien avec Christophe Chevalier le 20 novembre 2015.

${ }^{24} \mathrm{~L}$ 'Adié est une association reconnue d'utilité publique qui aide des personnes exclues du marché du travail et du système bancaire à créer leur entreprise et, donc, leur propre emploi, grâce au microcrédit.

${ }^{25} \mathrm{La}$ Nef est une banque alternative de la région lyonnaise créée en 1988, une coopérative financière qui offre des solutions d'épargne et de crédit orientées vers des projets ayant une utilité sociale, écologique et/ou culturelle.

${ }^{26}$ Fédération nationale de l'ESS.

${ }^{27}$ Sociétés coopératives de production.

${ }^{28}$ Société Coopérative d'Intérêt Collectif.

${ }^{29}$ Ces organismes sont : le Département de la Drôme, Région Rhône-Alpes, le Conseil Général de l'Ardèche, le Conseil Général de l'Isère, la Ville de Romans, la Ville de Bourg-de-Péage et la Communauté d'agglomération, la Direction générale des entreprises, de la concurrence et de la consommation, du travail et de l'emploi de RhôneAlpes, la Caisse des Dépôts et le Fonds social européen.

${ }^{30}$ Cet appel à projet est porté par un comité de pilotage réunissant le Secrétariat d'Etat au Commerce, à
l'Artisanat, à la Consommation et à l'Économie sociale et solidaire, le Ministère du Logement, de l'Egalité des territoires et de la Ruralité, le Ministère des Outre-mer, le Ministère de l'Agriculture, le Ministère de l'Éducation nationale, le Secrétariat d'État à la Politique de la Ville et la Caisse des Dépôts.

${ }^{31}$ Leur machine, L'Increvable, se veut être : réparable grâce à une accessibilité aisée aux pièces remplaçables, évolutive grâce à une architecture modulable, ouverte grâce à un logiciel en open-source et personnalisable grâce à une façade interchangeable (source : réunion du 2 juin 2016 au PTCE de Romans). L'équipe du PTCE leur propose de les aider à venir fabriquer leur prototype à Romans en les aidant à accéder à un local peu coûteux et en les mettant en relation avec des industriels du territoire. ${ }^{32} 115$ heures de formation réparties sur 4 mois seront dispensées à 12 futurs entrepreneurs. Les places seront proposées en priorité aux projets correspondant à la philosophie du projet «start up de terriroire ».

${ }^{33}$ Il s'agit bien d'accompagner les projets mais pas forcément de prendre des participations dans ces nouvelles entreprises (Conseil d'administration d'Archer du 6 juin 2016).

${ }^{34}$ Elle est soutenue par l'URSCOP, la COOPEA et la CRESS Rhône-Alpes.

${ }^{35}$ Source des informations : assemblée générale mixte de la SAS Groupe Archer du 6 juin 2016.

${ }^{36}$ VTD est spécialisée en outillage de découpe, mécanique générale de précision, fournitures pour outillages, découpe à façon jet d'eau et presse...

${ }^{37}$ Discussion avec Christophe Chevalier le 2 juin 2016.

${ }^{38} \mathrm{http}: / /$ www.milemil.fr/.

${ }^{39} \mathrm{La}$ logique d'Ulule n'est pas une logique d'investissement mais une logique de soutien, de don, ou de support à des projets spécifiques.

${ }^{40} 1083$ kilomètres, c'est la distance qui sépare les deux villes les plus éloignées l'une de l'autre en France, Menton (Var) et Porspoder (Finistère).

${ }^{41} \ll 1083$, le jean made in France, participatif et opensource », Florian Débes. Les Échos.fr.

${ }^{42}$ Depuis début août 2015, Le soulier français propose des services introuvables en France (développement des produits, recherche de fournisseurs, logistique...) et de la fabrication.

${ }^{43}$ Insoft est un atelier français de chaussures éco-conçues créé en 2011. Son siège social est à Châteauneuf sur Isère. ${ }^{44}$ Installée à l'origine près de Sisteron, la société a déménagé dans l'agglomération de Romans-sur-Isère en 2006. Elle produit des sandales en cuir.

${ }^{45}$ La maroquinerie proposée par Stéphanie Jimenez privilégie les fournisseurs locaux et est produite en petite série. ${ }^{46}$ Discussion avec la vendeuse du magasin Milémil le 2 juin 2016 à Romans-sur-Isère.

${ }^{47}$ Présentation de Christophe Chevalier, Assemblée générale mixte, SAS Groupe Archer, 6 juin 2016. 